\title{
Co-Surge Detection and Control for Bi-Turbo Engines with Experimental Evaluation
}

\author{
Andreas Thomasson* Lars Eriksson* \\ * Vehicular Systems, Dept. of Electrical Engineering \\ Linköping University, SE-581 83 Linköping, Sweden, \\ \{andreast,larer\}@isy.liu.se
}

\begin{abstract}
A V-type engine with a bi-turbocharger configuration utilizes the exhaust energy well which gives a fast torque response. An unwanted instability, called co-surge, can occur in such engines where the two interconnected compressors alternately go into flow reversals. If cosurge occurs, the control system must quell the oscillations with as little disturbance in engine torque as possible. A model of a bi-turbocharged engine is presented, combining a mean value engine model and a Moore-Greizer compressor model for surge. The model is validated against measurements on a vehicle dynamometer, showing that it captures the frequency and amplitude of the co-surge oscillation. The model is used to develop detection and control strategies for co-surge that rapidly returns the turbo to a stable operating point. Both simulations and experimental evaluation on the vehicle show that the developed strategies are successful in rapidly detecting and quelling co-surge. The selection of actuators is also studied. With no or small pressure drops over the throttle, it is necessary to use the bypass valves. However, for operating conditions with moderate and high pressure drops over the throttle, it is shown that it is sufficient to only open the throttle. This has the advantage, compared to opening the bypass valves, that it reduces the drop in boost pressure and thus reduces the drop in engine torque.
\end{abstract}

Keywords: Compressor surge, surge detection, surge control, engine modeling, engine control

\section{INTRODUCTION}

More advanced turbocharging concepts are constantly being developed to increase power density, and to reduce fuel consumption and emissions of internal combustion engines. For V-type engines one option is to use two smaller parallel turbos and let the exhausts from the two cylinder banks feed separate turbines. In this way the turbines can be placed closer to the exhaust ports than with a single larger turbo. Heat losses are reduced which increases the available energy to the turbochargers. With fewer cylinders feeding each turbine it is also possible to make better use of the pulsating flow, increasing the energy extracted from the exhaust.

In a bi-turbocharged engine the two air paths are connected before the throttle. If a disturbance alters the mass flow balance between them, when operating close to the surge line on an otherwise stable operating point, one compressor can be pushed into the surge region and the mass flow reverses. When the compressor recovers it can push the other compressor into surge, starting an oscillation where the mass flow through the compressors alternately reverses. This phenomenon with alternating flow reversals is called co-surge. Compressor surge should be avoided and thus needs to be considered when developing the control system for a bi-turbocharged engine.

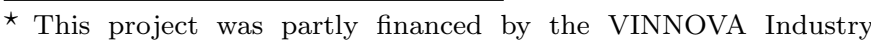
Excellence Center LINK-SIC.
}

\subsection{Contributions and Outline}

Compressor surge has been extensively studied during the 70's and 80's and a well known modeling result is the Moore-Greitzer model (Greitzer, 1981). The majority of the work has been on turbo machinery with gas turbines. A survey of surge modeling and control is given in Willems and de Jager (1998) and there is also a substantial treatment in Gravdahl (1998). For automotive turbochargers there are only a few studies on surge where most utilize the Moore-Greitzer model, see e.g. Ammann et al. (2001) or Leufven and Eriksson (2008). Studies of surge in bi-turbocharged engines are even scarcer, although the problem of one compressor pushing another into surge, when connected to a common intake, was pointed out by Watson and Janota (1982). The main contributions of this paper is an extension and experimental evaluation of the detection and control strategy for co-surge presented in Thomasson and Eriksson (2011). The paper strengthen the model validation from that paper with measurements from a vehicle dynamometer. With retuned turbo model parameters, correctly matched to the test vehicle, the agreement to measured data is significantly improved.

Section 2 presents the test vehicle and the experimental setup. Experimental data on co-surge and an analysis of the phenomenon is presented in section 3. Section 4 briefly outlines the model and evaluates it with new experimental data. A detection algorithm is outlined and evaluated in section 5 . In section 6 a control strategy to quell co-surge is presented. The controller is evaluated in a vehicle in a vehicle dynamometer and compared to simulation results. 


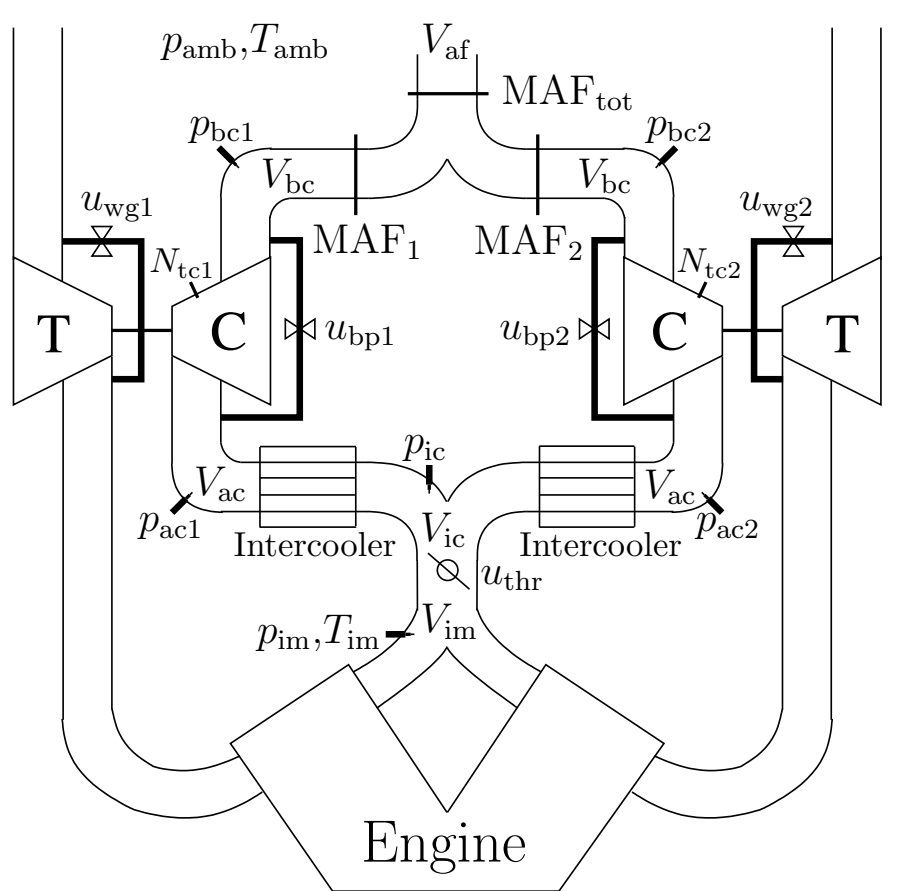

Fig. 1. A sketch of the bi-turbocharged engine configuration. A mass flow sensor, $\mathrm{MAF}_{\text {tot }}$, is positioned after the air filter and two more, $\mathrm{MAF}_{1}$ and $\mathrm{MAF}_{2}$, directly after the air path split up. The actuators used in the control section are the throttle, $u_{\mathrm{thr}}$, the bypass valves, $u_{\mathrm{bp} 1}, u_{\mathrm{bp} 2}$, and the wastegates, $u_{\mathrm{wg} 1}, u_{\mathrm{wg} 2}$ Pressures are measured before and after each compressor, before the throttle and in the intake manifold.

\section{TEST SETUP}

The test vehicle is equipped with a gasoline V6-engine with two parallel turbochargers, each powered from one bank of cylinders. A sketch of the engine is shown in Figure 1, that also defines the nomenclature which is also available in Appendix A. The engine is equipped with three hot film mass flow sensors that are placed approximately $50 \mathrm{~cm}$ after the air filter, $80 \mathrm{~cm}$ before the compressors, one directly before and two directly after the air path is divided. Pressures are measured before and after each compressor, before the throttle and in the intake manifold. The turbochargers are equipped with speed sensors. The measurement and control system is a dSpace MicroAutoBox and a RapidPro system, connected to a computer running ControlDesk. The actuators used by the control algorithm are the throttle, the bypass valves and the wastegates. The throttle and wastegate are continuously actuated while the bypass valves are of $\mathrm{ON} / \mathrm{OFF}$ type. For the tests the vehicle is mounted in a vehicle dynamometer with one electric motor connected to each wheel on the rear axis. Although the test vehicle is equipped with lots of sensors for modeling, only the two mass flow sensors, $\mathrm{MAF}_{1}$ and $\mathrm{MAF}_{2}$, the pressure after the intercooler, $p_{\text {ic }}$, and the intake manifold pressure, $p_{\text {im }}$, are used in the detection and control algorithms.

\section{CO-SURGE}

An example of co-surge measured in a test vehicle will be shown in Figure 3. (The figure is placed in the model section for convenience of validation.) The mass flow balance between the two air paths is slightly unbalanced.
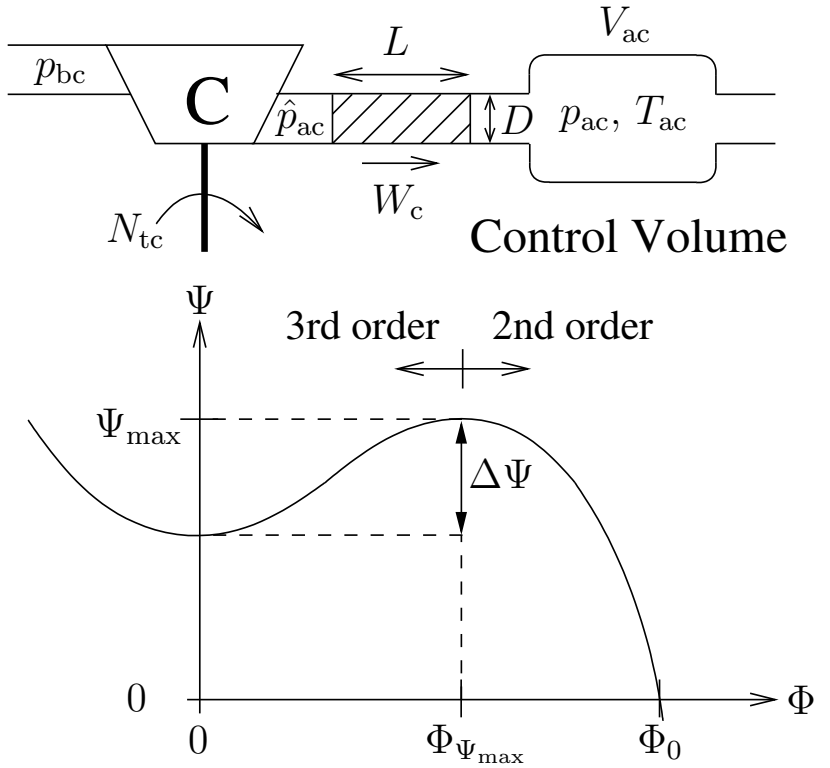

Fig. 2. Top: The Moore-Greitzer compressor model. The pressure difference $\hat{p}_{\mathrm{ac}}-p_{\mathrm{ac}}$ results in an acceleration of the flow plug that governs the mass flow.

Bottom: Parameterization of the $\Phi-\Psi$ function.

After a small decrease in mass flow, one compressor flow reverses, starting an oscillation between the compressors. Surge occurs when the pressure ratio is too high for the current mass flow to be maintained. When the compressor enters this region the mass flow will start to reverse. The flow is not recovered until the pressure ratio has decreased sufficiently. Co-surge is a condition in the bi-turbo configuration, where the mass flow through the compressors alternately reverses. When one compressor enters surge more air will flow through the other compressor due to the pressure ratio decrease. The compressor that has entered surge will speed up since it no longer consumes any work, as opposed to the other compressor which slows down. Thus the oscillation is also present in the compressor speed but with opposite phase compared to the mass flow. When the first compressor recovers the second compressor is pushed into surge. Compared to normal surge, co-surge has a much lower frequency, roughly one order of magnitude. This indicates that co-surge is more than standard compressor surge with alternating flow reversals.

\section{ENGINE MODEL}

To efficiently and safely test new control strategies it is advantageous to use a model of the system before testing in a real environment. To do this a model that can capture the most important system properties, in this case cosurge, is needed. The model was outlined in Thomasson and Eriksson (2011) and in this paper the model validation is strengthened with additional measurements including turbocharger speed.

The modeling approach taken is the component based Mean Value Engine Model (MVEM) outlined in Eriksson et al. (2002); Eriksson (2007). A complete turbocharged spark ignited engine with these components is implemented and evaluated in Andersson (2005). For this investigation the MVEM components have been arranged in a bi-turbo structure to resemble the engine in Figure 1. 

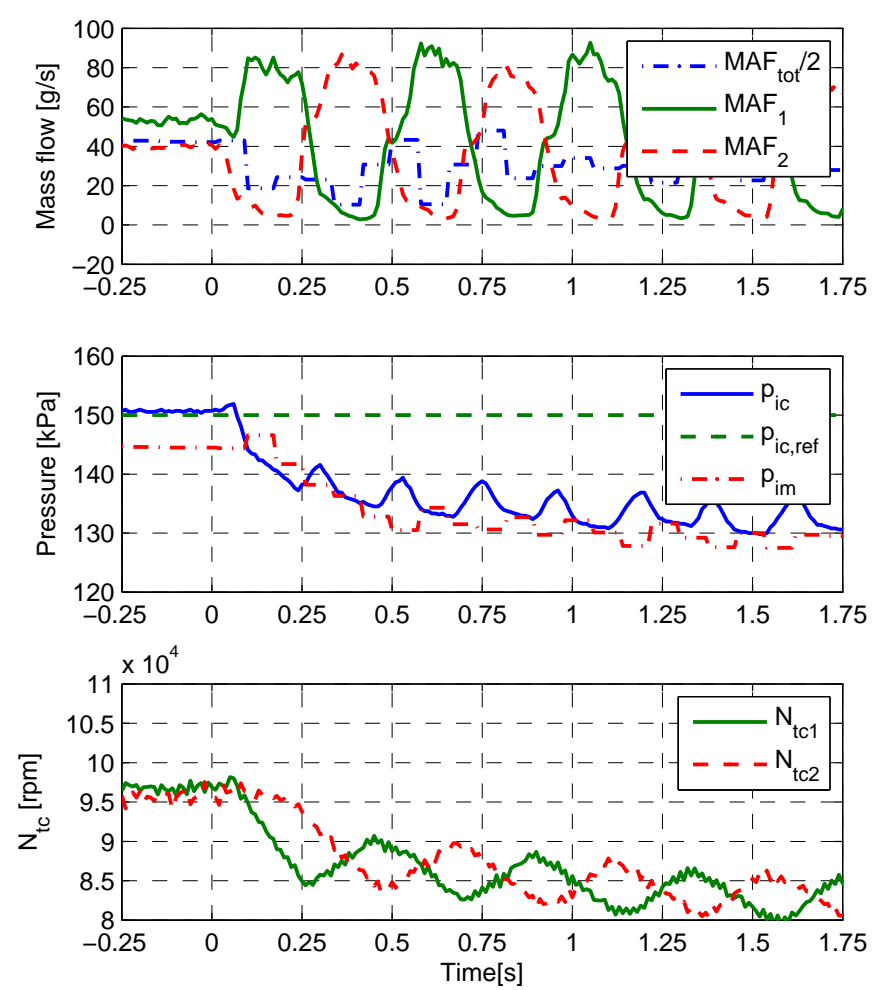

Fig. 3. An example of co-surge measured in test vehicle. The mass flows are imbalanced when a small disturbance occur at $\mathrm{t}=0 \mathrm{~s}$. The mass flows rapidly diverge and an oscillation starts. The oscillation is present in the turbo speed but opposite phase to the mass flow.

\subsection{Compressor model}

To model surge the compressor model must handle reverse mass flow. This is achieved by the well known and well tested Moore-Greizer model (Greitzer, 1981), that incorporates an additional state for the mass flow, $W_{\mathrm{c}}$.

$$
\frac{d W_{\mathrm{c}}}{d t}=\frac{\pi D^{2}}{4 L}\left(\hat{p}_{\mathrm{ac}}-p_{\mathrm{ac}}\right)
$$

The difference between pressure build up, $\hat{p}_{\text {ac }}$, and pressure after the compressor, $p_{\text {ac }}$, results in a force that accelerates a flow plug, with length $L$ and diameter $D$, that govern the mass flow, see Figure 2. This model was first developed for axial flow compressors in Greitzer (1976), and was shown to work for centrifugal compressors in Hansen et al. (1981).

In the model it is necessary to have a description of the compressor speed lines. Here a simple parametrization is used, based on the dimensionless quantities for flow $\Phi$ and energy $\Psi$. They are defined as (Dixon, 1998)

$$
\begin{gathered}
\Phi=\frac{W_{\mathrm{c}}}{N D^{3}} \frac{R T_{\mathrm{bc}}}{p_{\mathrm{bc}}} \\
\Psi=\frac{c_{\mathrm{p}} T_{\mathrm{bc}}\left(\Pi_{\mathrm{c}}^{(\gamma-1) / \gamma}-1\right)}{N^{2} D^{2}}
\end{gathered}
$$

where $D$ is the compressor diameter, $N$ is the rotational speed, $T_{\mathrm{bc}}$ is the temperature before the compressor, $\mathrm{R}$ is the specific gas constant for air and $\Pi_{\mathrm{c}}=\hat{p}_{\mathrm{ac}} / p_{\mathrm{bc}}$. When transformed into the $\Phi-\Psi$ domain, the speed lines in the compressor map gathers into almost a single curve (Eriksson, 2007). The model uses the relation between $\Phi$ and $\Psi$ to span the compressor map and it is represented by the combination of a third and a second order polynomial.
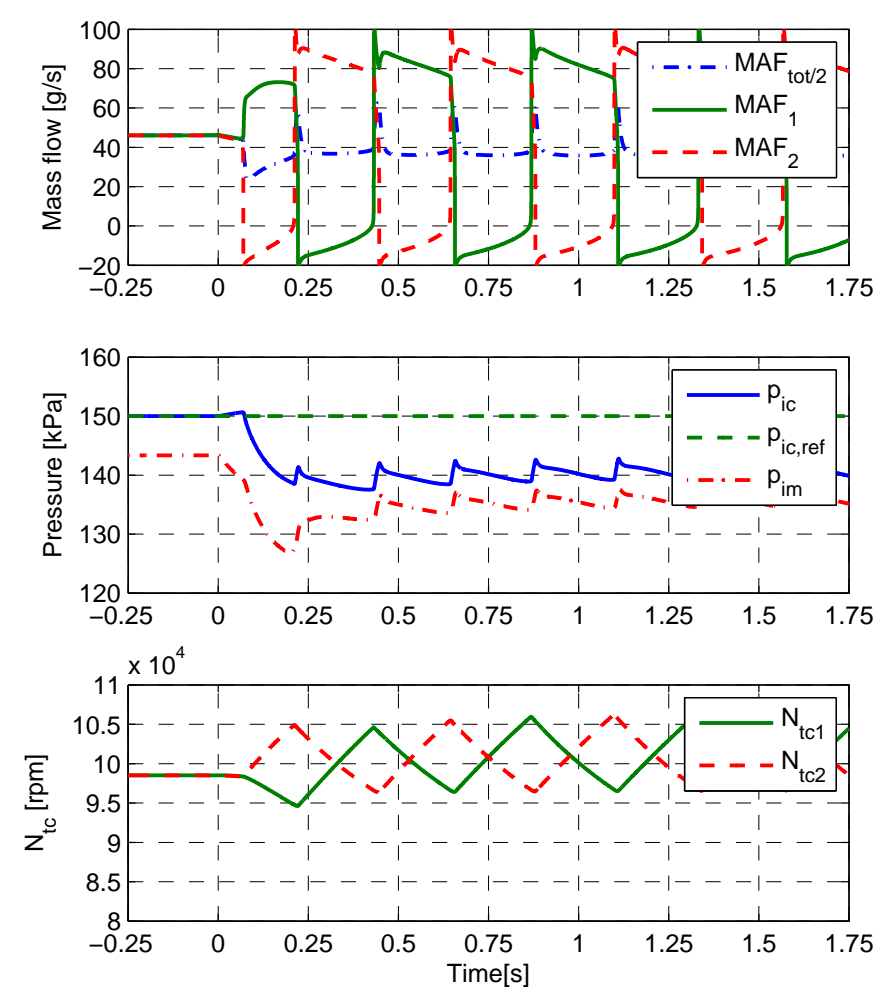

Fig. 4. Co-Surge simulation with the developed model. Both the amplitude and frequency for the mass flow and pressure oscillations is similar to the measured data. The oscillation in compressor speed is slightly larger than the in the measurements and the initial drop in speed is not present in the simulation.

$$
\Psi(\Phi)= \begin{cases}a_{3} \Phi^{3}+a_{2} \Phi^{2}+a_{1} \Phi+a_{0} & \text { if } \Phi \leq \Phi_{\Psi_{\max }} \\ b_{2} \Phi^{2}+b_{1} \Phi+b_{0} & \text { if } \Phi>\Phi_{\Psi_{\max }}\end{cases}
$$

The parameters $a_{i}$ and $b_{i}$ in $\Psi(\Phi)$ are determined from the parameters $\Psi_{\max }, \Delta \Psi, \Phi_{\Psi_{\max }}$ and $\Phi_{0}$, together with the constraints $\Psi^{\prime}(0)=0$ and $\Psi^{\prime}\left(\Phi_{\Psi_{\max }}\right)=0$, see Figure 2 . For this investigation, measurements on co-surge has been made in high load operating points close to the surge line around $\Pi_{c}=1.5$. The model parameters have been tuned for best fit in this region. A study of how the model parameters affect the co-surge behavior was presented in Thomasson and Eriksson (2011).

\subsection{Model validation}

Figures 3 and 4 compare measurement and simulation for a constant operating point, where a small throttle disturbance at $\mathrm{t}=0 \mathrm{~s}$ results in co-surge. The amplitude and frequency of the mass flow and pressure oscillations is similar to the measured data. The model shows the same behavior in turbo speed, an oscillation with opposite phase compared to the mass flow. The model shows negative mass flow during the surge period but the mass flow sensors can not measure negative flows. However, during cosurge the measured mass flow from the side with positive flow is larger then the measured total mass flow. The only explanation for this is that air flows backward from the other side. A major difference is that the boost pressure in the measurement initially drops down to the intake manifold pressure, whilst in the simulation both intake manifold an boost pressure drops simultaneously. As a 

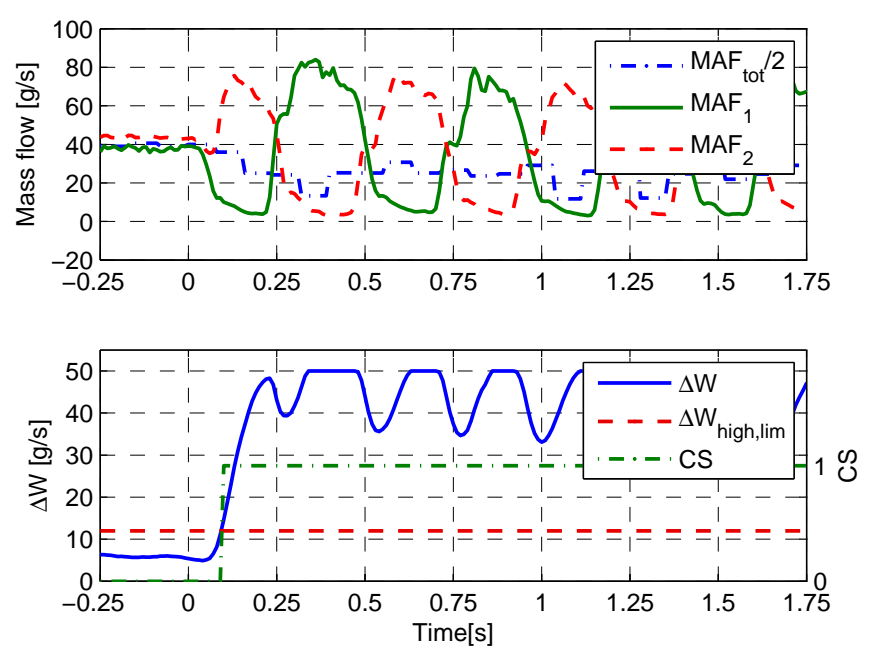

Fig. 5. An example of co-surge detection where a disturbance has been introduced at $\mathrm{t}=0 \mathrm{~s}$. The top figure shows the mass flows which start to diverge approximately $0.05 \mathrm{~s}$ after the disturbance. The bottom figure shows the filtered difference $\Delta W$, the threshold $\Delta W_{\text {high,lim }}=12 \mathrm{~g} / \mathrm{s}$ and the CS variable, that indicate that co-surge is detected after $0.1 \mathrm{~s}$

result, the boost pressure in the measurement equilibrates periodically. The transition between positive and negative flow is faster in the model, but no sensor dynamics or flow inertia effects are included. A slow decay in turbo speed is present in the measurement, possibly due to increased friction during surge. To summarize, although there are slight differences, the quantitative properties, the amplitude of the mass flow, pressure and turbo speed oscillation, the oscillation frequency and the opposite phase of the mass flow and turbo speed are captured by the model.

\section{DETECTION}

For the detection algorithm it is assumed that one mass flow sensor in each air path is available. When co-surge begins the mass flow in the two air paths diverge rapidly. The detection is therefore based on the difference in mass flow between the two air paths. The absolute value of the difference is low-pass filtered to remove high frequency disturbance, but with a cut-off frequency high enough for fast detection. The filtered difference, $\Delta W$, is used to determine if the system has entered co-surge. When $\Delta W$ exceeds the threshold $\Delta W_{\text {high,lim }}$, the variable CS is set to one, indicating that co-surge has been detected. The variable $\Delta W$ is saturated at 50 to quicken the response when co-surge ends, and hysteresis, with the lower limit $\Delta W_{\text {low,lim }}$, is used to avoid switching during transitions.

$$
\begin{aligned}
\Delta W & =\frac{1-k}{1-k z^{-1}}\left|\mathrm{MAF}_{1}-\mathrm{MAF}_{2}\right| \\
\mathrm{CS} & = \begin{cases}1, & \text { if } \Delta W \geq \Delta W_{\text {high,lim }} \\
1, & \text { if } \Delta W \geq \Delta W_{\text {low,lim }} \text { and } z^{-1} \mathrm{CS}=1 \\
0, & \text { otherwise }\end{cases}
\end{aligned}
$$

The variable $z$ is the z-transform operator and $k$ depends on the sample rate and desired cut-off frequency for the low-pass filter. The threshold $\Delta W_{\text {high,lim }}$ must be larger than the mass flow difference expected during normal operation due to disturbances etc. In Figure 5 the detection is
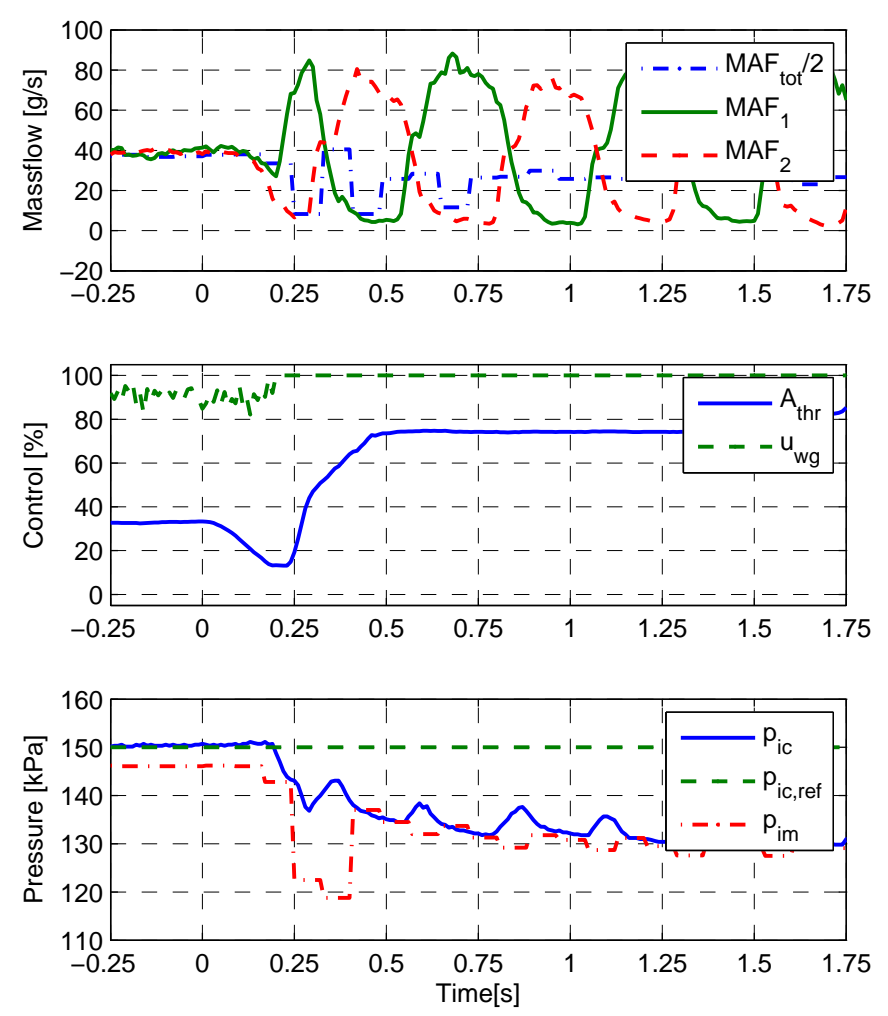

Fig. 6. Example of when opening the throttle is not enough to quell the co-surge oscillation. In this case the pressure drop over the throttle before the oscillation is approximately $4 \mathrm{kPa}$. Co-surge is triggered by a short throttle closing at $\mathrm{t}=0 \mathrm{~s}$. When co-surge is detected the throttle opens but the oscillation continues.

evaluated on measurements from the test vehicle. The evaluation runs at $\mathrm{MAF}_{\text {tot }} \approx 80 \mathrm{~g} / \mathrm{s}$ with $\Delta W_{\text {high,lim }}=12 \mathrm{~g} / \mathrm{s}$, $15 \%$ of the total mass flow. A disturbance is introduced at $\mathrm{t}=0 \mathrm{~s}$, after about $0.05 \mathrm{~s}$ the mass flows start to diverge and co-surge is detected at $\mathrm{t}=0.1 \mathrm{~s}$.

\section{CONTROL}

There are several methods for avoiding or suppress surge in single compressors described in the literature (de Jager, 1995). In active surge control the operating region of the compressor is extended by stabilizing the compressor in the otherwise unstable region to the left of the surge line, see for example Willems and de Jager (1998); Gravdahl and Egeland (1999). In surge avoidance strategies the compressor is controlled to operate with a safety margin to the surge line in order to avoid surge Gravdahl (1998). Surge detection an avoidance strategies tries to avoid the drawbacks of operating with a safety margin by detecting the onset of surge and then act to move the operating point away from the unstable region de Jager (1995). Co-surge is partly a different control problem. When cosurge occurs due to a disturbance between the two mass flows, at a constant operating point, the operating point with balanced mass flows is stable. The objective of the control system is then to balance the flow and return to this operating point, as fast and with as little torque disturbance as possible.

The actuators that rapidly can change the compressor operating conditions are the throttle and bypass valves. 

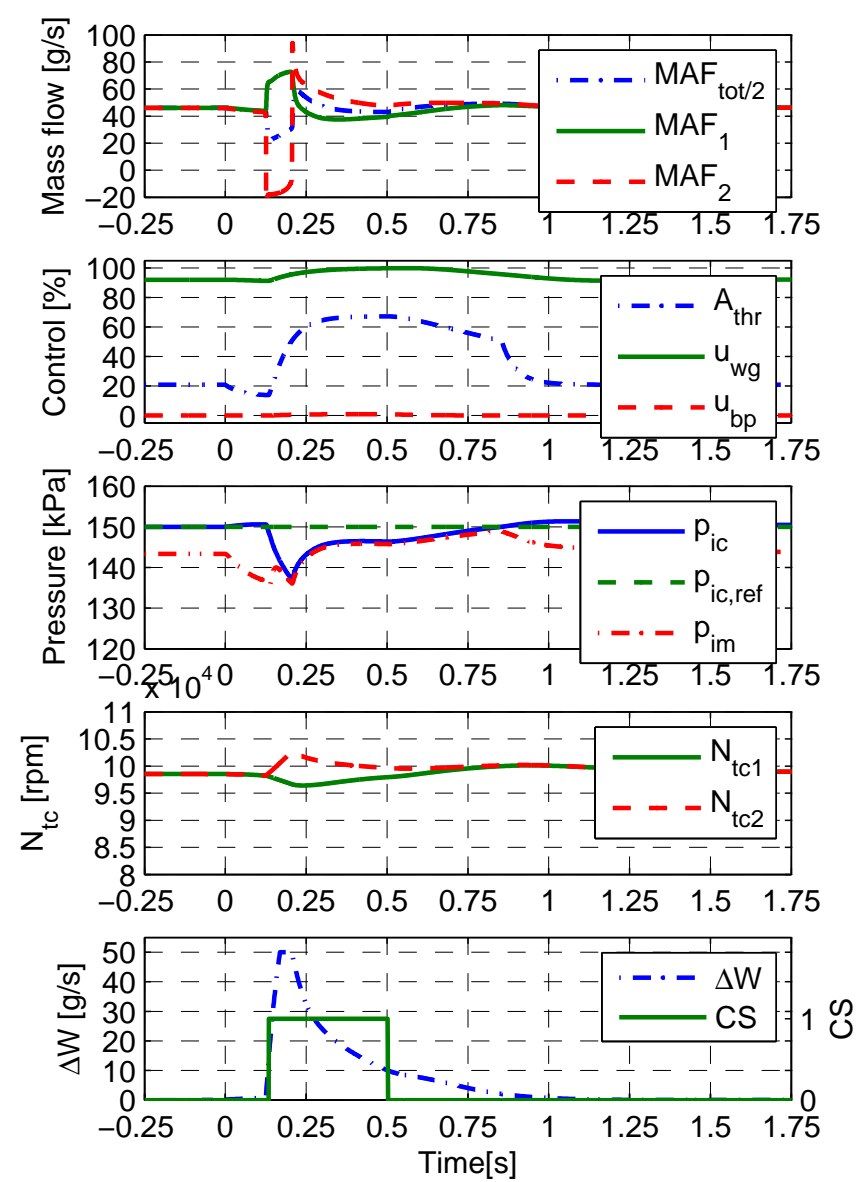

Fig. 7. Evaluation of the stabilization method on the model. At $\mathrm{t}=0 \mathrm{~s}$ a disturbance alters the mass flow balance, pushing one flow into reverse. When this is detected, the throttle is opened up and stable operation is recovered in about $0.5 \mathrm{~s}$.

Opening the throttle reduces the boost pressure for a given mass flow, moving the operating point to the right in the compressor map and increasing the surge margin. The mass flow will also increase, partly counteracting the reduction in total mass flow that occurs during cosurge. Opening the bypass valves reduces the pressure ratio and increases the mass flow felt by the compressor by recirculating part of the compressed air. This moves the operating point of the compressor away from the surge region but also reduces boost pressure and thus leading to greater torque disturbance.

To minimize torque disturbance and the time to recover from co-surge it is therefore beneficial to open the throttle and keep the bypass valves closed, if this is enough to quell the oscillation. Several experiments where made for different operating points to investigate when this is possible. Co-surge was initialized by a short throttle disturbance for otherwise constant operating conditions. The result correlates well to when the throttle has authority, i.e. when there is a pressure drop over the throttle. When the pressure drop over the throttle is larger than $10 \mathrm{kPa}$, throttle actuation alone was always able to quell the oscillation. For $5-10 \mathrm{kPa}$ the oscillation was quelled in most cases, with lower success rate for lower pressure drops. If the pressure drop over the throttle is too small, the bypass valves need to be opened. An example of when opening
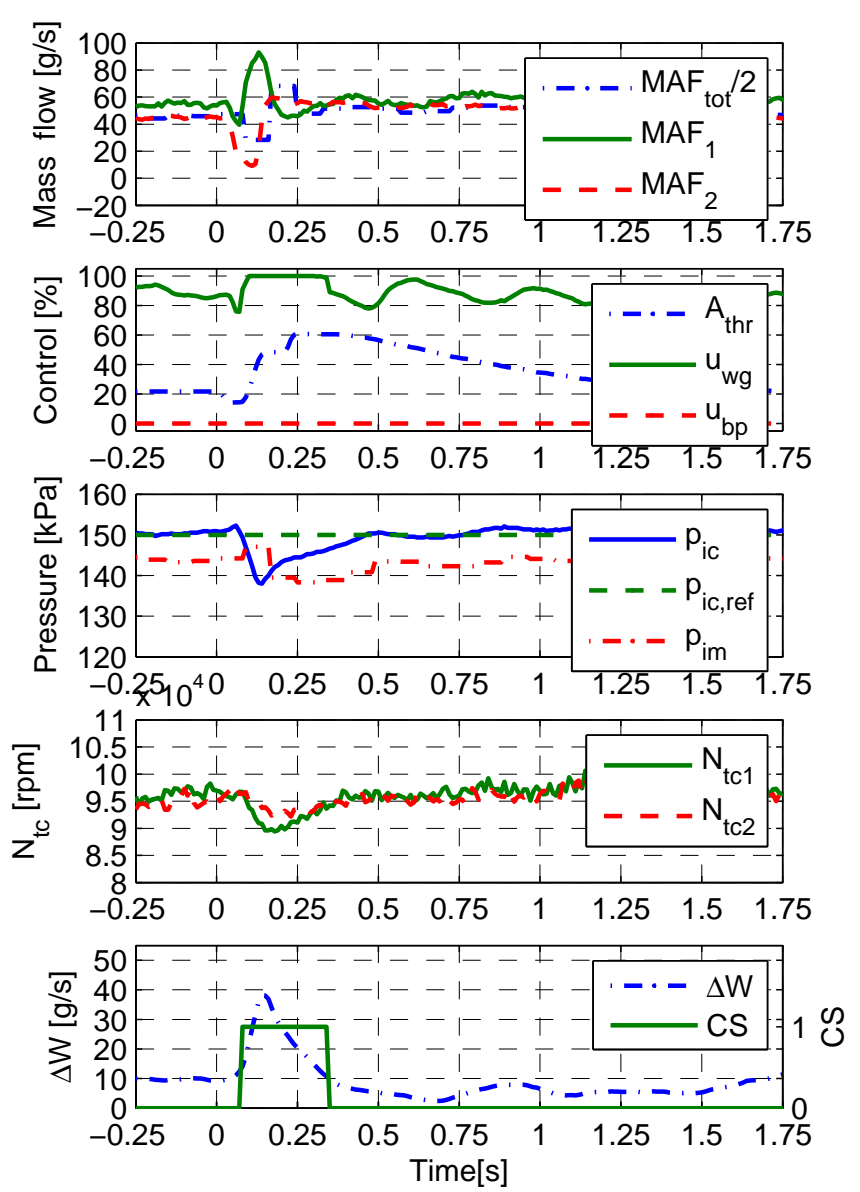

Fig. 8. Evaluation of the co-surge control strategy for a constant operating point. At $\mathrm{t}=0 \mathrm{~s}$ a disturbance is introduced resulting in co-surge. The controller opens the throttle and recovers stable operation in $0.5 \mathrm{~s}$.

the throttle is not enough is shown in Figure 6. Co-surge is induced by a short closing of the throttle at $t=0 \mathrm{~s}$. Due to the pressure drop when the system enters co-surge the boost controller closes the wastegates (100\% control signal is commanded). When the oscillation starts and co-surge is detected, the throttle is opened up but the oscillation continues undamped.

The control strategy proposed here is therefore to only open the throttle and keep the bypass valves closed if the pressure drop over the throttle, $\Delta p_{\mathrm{thr}}$, is above a certain limit, $\Delta p_{\text {lim }}$, provided that the desired boost pressure and intake manifold pressure is below their respective reference values. If the pressure drop is lower, and if intake manifold pressure is below the reference, both throttle and bypass valves are opened. Otherwise only the bypass valves are opened. This is written in equation form as

$$
\begin{aligned}
& \Delta u_{\mathrm{thr}}= \begin{cases}k_{\mathrm{thr}}, & \text { if } \mathrm{CS}=1 \text { and } p_{\mathrm{im}}<p_{\mathrm{im}, \mathrm{ref}} \\
\Delta u_{\mathrm{thr}}(k-1)-k_{\mathrm{thr}} \frac{\tau_{\mathrm{s}}}{\tau_{\mathrm{thr}}}, \quad \text { if } \mathrm{CS}=0\end{cases} \\
& u_{\mathrm{bp}}= \begin{cases}1, & \text { if } \mathrm{CS}=1 \text { and } \Delta p_{\mathrm{thr}}\left(\mathrm{t}_{\mathrm{CS}}\right)<\Delta p_{\mathrm{lim}} \\
1, & \text { if } \mathrm{CS}=1 \text { and } p_{\mathrm{im}} \geq p_{\mathrm{im}, \mathrm{ref}} \\
0, & \text { otherwise }\end{cases}
\end{aligned}
$$

where $\Delta u_{\mathrm{thr}}$ is an additive throttle reference, $u_{\mathrm{bp}}$ is bypass valve command, $(k-1)$ indicate previous sample, $t_{\mathrm{CS}}$ is the time when co-surge was detected (the last time 
sample when CS went from zero to one), $k_{\text {thr }}$ is the amplitude of throttle opening, $\tau_{\mathrm{s}}$ is the sample time and $\tau_{\text {thr }}$ determines how fast the throttle is ramped down. This works in parallel with a standard boost pressure controller, consisting of a gain scheduled PID-controller and a feedforward, that commands the two wastegates.

\subsection{Evaluation}

The control strategy is first tested in simulation environment. A simulation with a constant operation point can be seen in Figure 7. At $\mathrm{t}=0 \mathrm{~s}$ a small disturbance results in co-surge. The control system detects the divergence in mass flow at $\mathrm{t}=0.13 \mathrm{~s}$ and opens the throttle. The mass flow quickly recovers and after about $0.75 \mathrm{~s}$ boost pressure is back to the same level as before the disturbance.

The control strategy was then implemented in a test vehicle. Figure 8 show one experiment with the suggested control strategy. At $\mathrm{t}=0 \mathrm{~s}$ a small disturbance in throttle position is causing the mass flows to diverge. The difference in mass flow is detected and since $\Delta p_{\text {thr }}$ is high enough and $p_{\mathrm{bc}}$ is below the reference, the throttle is opened. The mass flow balance is quickly restored and mass flow and pressure is back to level within $0.5 \mathrm{~s}$. Comparing the simulation result and the experimental data the behavior in both mass flow and pressure are similar. The recovery in the simulation is slightly slower, probably because the positive peak in $\mathrm{MAF}_{2}$ is not present in the measurement. As shown earlier the initial speed drop in turbocharger speeds is not present in the simulation, but the amplitude of the divergence is similar as in the measurements.

\section{CONCLUSIONS}

Measurements of co-surge from a test vehicle with a biturbocharged engine, in a vehicle dynamometer, is presented. The paper presents an engine model capable of capturing co-surge. This is achieved by combining a mean value engine model with a Moore-Greitzer compressor model. The model is compared to experimental data and is shown to capture the qualitative properties of co-surge, the amplitude and frequency of the pressure and mass flow oscillation and the turbo speed oscillation with opposite phase compared to the mass flow. A detection algorithm to rapidly detect co-surge is presented and validated against measured data. A control strategy is presented that aims to quell the co-surge oscillation with as little torque disturbance as possible. The experimental evaluation shows that the the co-surge oscillation is quenched and the preceding operating point is recovered in $0.5 \mathrm{~s}$. Additionally there is good agreement between simulations and experiments with the suggested controller structure, further strengthening the model validity.

\section{REFERENCES}

Ammann, M., Fekete, N.P., Amstutz, A., and Guzzella, L. (2001). Control-oriented modeling of a turbocharged common-rail diesel engine. In Proceedings from the $3 \mathrm{rd}$. Int. Conference on Control and Diagnostics in Automotive Applications (CD Auto '01), Sestri Levante.

Andersson, P. (2005). Air Charge Estimation in Turbocharged Spark Ignition Engines. Ph.D. thesis, Linköping University. de Jager, B. (1995). Rotating stall and surge control: A survey. In Proceedings of the 34th IEEE Conference on Decision and Control, volume 2, 1857-1862.

Dixon, S. (1998). Fluid Mechanics and Thermodynamics of Turbomachinery. Butterworth-Heinemann, 4th edition.

Eriksson, L. (2007). Modeling and control of turbocharged SI and DI engines. Oil $\&$ Gas Science and Technology Rev. IFP, 62(4), 523-538.

Eriksson, L., Nielsen, L., Brugård, J., Bergström, J., Pettersson, F., and Andersson, P. (2002). Modeling of a turbocharged SI engine. Annual Reviews in Control, 26(1), 129-137.

Gravdahl, J.T. (1998). Modeling and Control of Surge and Rotating Stall in Compressors. Ph.D. thesis, Norweigan University of Science and Technology.

Gravdahl, J.T. and Egeland, O. (1999). Centrifugal compressor surge and speed control. Transactions on Control Systems Technology, 7(5), 567-579.

Greitzer, E. (1976). Surge and rotating stall in axial flow compressors-part I: Theoretical compression system model. Journal of Engineering for Power, 98(2), 190198.

Greitzer, E. (1981). The stability of pumping systems. Journal of Fluids Engineering, Transactions of the ASME, 103(1), 193-242.

Hansen, K., Jørgensen, P., and Larsen, P. (1981). Experimental and theoretical study of surge in a small centrifugal compressor. Journal of Fluids Engineering, Transactions of the ASME, 103, 391-395.

Leufven, O. and Eriksson, L. (2008). Time to surge concept and surge control for acceleration performance. In IFAC World Congress. Seoul, Korea.

Thomasson, A. and Eriksson, L. (2011). Modeling and control of co-surge in bi-turbo engines. In IFAC World Congress. Milano, Italy.

Watson, N. and Janota, M. (1982). Turbocharging the Internal Combustion Engine. The Macmillan Press ltd. ISBN 0-333-24290-4.

Willems, F. and de Jager, B. (1998). Modeling and control of rotating stall and surge: An overview. In Int. Conference on Control Applications, 331-335.

Appendix A. NOMENCLATURE

\begin{tabular}{lr||lr}
\hline Symbol & Description & Subscript & Description \\
\hline$A$ & Area & af & Air filter \\
$c_{\mathrm{p}}$ & Specific heat & bc & Before compressor \\
$D$ & Diameter & bp & Bypass \\
$\gamma$ & $c_{\mathrm{p}} / c_{\mathrm{V}}$ & $\mathrm{CS}$ & Co-surge detected \\
$L$ & Flow plug length & $\mathrm{c}$ & Compressor \\
$\mathrm{MAF}$ & Mass flow sensor & fric & Friction \\
$N$ & Rotation speed & ic & Intercooler \\
$\Pi$ & Pressure ratio & im & Intake manifold \\
$p$ & Pressure & lim & Limit \\
$\Delta p$ & Pressure difference & ref & Reference \\
$R$ & Gas constant & $\mathrm{t}$ & Turbine \\
$T$ & Temperature & tc & Turbocharger \\
$\mathrm{t}$ & Time & thr & Throttle \\
$\tau$ & Time constant & & \\
$u$ & Control signal & & \\
$V$ & Volume & & \\
$W$ & M-transform operator & & \\
$\omega$ & Angular velocity & & \\
$z$ & Co-surge detected & & \\
\hline
\end{tabular}

